\title{
Elastic interaction and superstructures in manganites and other Jahn-Teller systems
}

\author{
D. I. Khomskii ${ }^{a}$ and K. I. Kugel ${ }^{b}$ \\ ${ }^{a}$ Laboratory of Solid State Physics, Groningen University, \\ Nijenborgh 4, 9747 AG Groningen, The Netherlands \\ ${ }^{b}$ Institute for Theoretical and Applied Electrodynamics, Russian Academy of Sciences, \\ Izhorskaya Str. 13/19, 127412 Moscow, Russia
}

(October 27, 2018)

\begin{abstract}
The role of elastic interactions between Jahn-Teller ions in formation of various orbital- and chargeordered structures in manganites and related compounds is analyzed. It is shown that such interactions alone are often sufficient to reproduce the structures observed in different regions of the phase diagram. A special attention is focused on stripe structures at high doping levels.
\end{abstract}

PACS: 64.75.+g, 61.50.Ah, 71.28.+d

\section{INTRODUCTION}

As is well established now on many examples, an orbital degeneracy and Jahn-Teller effect play very important role in various transition-metal and rare-earth compounds [1]. In concentrated systems, it leads typically to lowering of lattice symmetry, often taking the form of a structural phase transition; it is associated with (or caused by) the orbital ordering. Correspondingly, also magnetic properties are largely determined by the type of occupied orbitals according to the GoodenoughKanamori-Anderson rules, see e.g. [1, 4,5].

Manganites $\mathrm{La}_{1-x} M_{x} \mathrm{MnO}_{3}(M=\mathrm{Ca}, \mathrm{Sr}, \mathrm{Ba})$ with the colossal magnetoresistance (CMR) form a very popular nowadays system, for which the orbital effects often play very important role. There may be other rare earths (Pr, $\mathrm{Nd}, \mathrm{Sm}$ ) instead of La in these series; there exist also layered analogues of three-dimensional perovskite manganites, often having similar properties. It is established that there exists an orbital ordering of some kind in almost all parts of the phase diagram of these systems. Thus, it exists in $\mathrm{La}_{1-x} \mathrm{Ca}_{x} \mathrm{MnO}_{3}$ at $x=0$; probably in the charge ordered insulating phases at small $x(x \sim 0.1-0.2)$; at the checkerboard-like charge ordered phase at $x=0.5$ (so called CE phase); in the overdoped regimes $0.5 \leq x \leq 1$, where charge and orbital stripes [6, 7] or bistripes [8] were observed. The only exception may be the "optimally doped" ferromagnetic metallic phase $0.25 \leq x \leq 0.5$, where the very phenomenon of CMR is taking place: no apparent orbital ordering of usual type is observed there at low temperatures [9] although the possibility of an orbital ordering of a new type (with "complex orbitals") was suggested even for this phase [10]. In the present paper, we will not discuss these more exotic possibilities and will limit ourselves to the conventional types of orbital ordering with real orbitals.

There exist in principle two different mechanisms of orbital ordering, or cooperative Jahn-Teller effect. First, it is the usual interaction of degenerate electrons with the crystal lattice [2,11,12], which is usually considered as a source of the Jahn-Teller effect. In concentrated systems, this interaction provides the coupling of orbital occupation at different sites and can lead to an orbital ordering simultaneously with the corresponding lattice distortion. The second mechanism is the electronic (exchange) one 113,3, which can give rise to both the spin and orbital orderings, and which was quite successful in explaining the magnetic and orbital structures in a number of materials, notably cuprates [3].Most of the previous theoretical considerations were confined to undoped systems containing integer number of electrons at the transition metal sites: $d^{9}$ for $\mathrm{Cu}^{2+}, d^{4}$ for $\mathrm{Mn}^{3+}$. An important class of systems is represented by the doped oxides like $\mathrm{La}_{1-x} \mathrm{Ca}_{x} \mathrm{MnO}_{3}$, where, as mentioned above, similar phenomena of orbital ordering are also often observed. One should generalize the models used for undoped systems to such cases, in particular to be able to explain different superstructures observed in these systems, e.g., stripes or paired stripes (bistripes).

Such treatment was recently initiated in [14, where we considered the formation of superstructures, including stripes, due to elastic interaction, when we dope our system, i.e. substitute ions of one valence (and one size) by an "impurity" with the different valence and different atomic volume. It was shown there that due to a specific nature of these interactions (long-range character, $\sim 1 / R^{3}$, and, most importantly, different sign - repulsion or attraction - in different directions) the structures of different kinds - 1D stripes, 2D "sheets" - can be naturally formed in these cases.

When considering the case of Jahn-Teller (JT) systems, one should modify this treatment by taking into account the anisotropic nature of corresponding electronic states, i.e. anisotropic electronic charge distribution(having quadrupolar character) and, correspondingly, the shape of a Jahn-Teller ion in a respective orbital state. Thus, in contrast to the treatment of [14], we have to consider not the interaction of spherical impurities ("sphere-in-the-hole" model) but rather that of anisotropic impurities ("ellipsoid-in-the-hole"). This 
generalization was shortly mentioned at the end of [14]; in the present paper, we consider this situation in detail and discuss the applications to the superstructures observed in manganites in different regions of the phase diagram. As we shall see, in some cases such interactions alone are sufficient to reproduce the observed structures. In other situations, some extra factors have to be invoked, but in any case one can say that the elastic interactions considered in this paper are definitely very important in stabilizing the observed orbital and, consequently, magnetic structures.

\section{STRAIN MECHANISM OF SUPERSTRUCTURE FORMATION}

First, we shortly summarize the results concerning the ordering of "spherical" impurities due to elastic forces [14]. When we put into the host matrix an ion of different size, e.g. by substituting an ion with the atomic volume $v_{0}$ by an impurity with the volume $v \neq v_{0}$, this creates a strain field, which decays rather slowly, as $1 / R^{3}$ [15]. Another such impurity interacts with this strain, which therefore leads to a certain coupling between impurities. If both the host and impurity ions are spherical, this is what is called "sphere-in-the-hole" model. It is known that the strain-induced interaction vanishes for the isotropic media [15] (except of the infinite-range interaction caused by the "mirror forces" related to the sample surface). However, the real crystals are always anisotropic. This gives rise to an interaction, which has the following form for weakly anisotropic cubic crystals [14, 15:

$$
V\left(\mathbf{r}, \mathbf{r}^{\prime}\right)=-\frac{C Q_{1} Q_{2} d\left(n_{x}^{4}+n_{y}^{4}+n_{z}^{4}-\frac{3}{5}\right)}{R^{3}}
$$

Here $Q_{i}=v_{i}-v_{0}$ are the "strengths" of the impurities, $R=\left|\mathbf{r}-\mathbf{r}^{\prime}\right|$ is the distance between them, $n_{x}, n_{y}$, and $n_{z}$ are the direction cosines of vector $\mathbf{R}$. The most important parameter entering (1) is

$$
d=c_{11}-c_{12}-2 c_{44}
$$

where $c_{i j}$ are elastic moduli of the crystal. This parameter carries the information about anisotropy: the case $d=0$ corresponds to an isotropic medium.

The most significant feature of the interaction (1) is that it is attractive in certain directions independent of the impurity type $\left(v>v_{0}\right.$ or $\left.v<v_{0}\right)$ and of the sign of the coefficient $d$. Thus for $d>0$ the interaction is attractive along cubic axes [100], [010], and [001] and repulsive along face and body diagonals of the cubic cell, [110] and [111], and vice versa for $d<0$. As argued in [14], this quite naturally leads to the formation of superstructures (1D stripes, 2D sheets) in insulating systems: the second, third, etc. impurities "migrate" toward the first one along certain directions, e.g. [001] for $d>0$, and finally form vertical (diagonal for $d<0$ ) stripes along this direction. Important is also that here this motion of impurities is provided by the electron hopping and does not require real diffusion of atoms; thus, e.g., holes moving in nickelates transform $\mathrm{Ni}^{2+}$ ions into $\mathrm{Ni}^{3+}$ "impurities", which can form stripes due to this mechanism.

When considering such processes in systems with JahnTeller ions, e.g. in manganites, one has to generalize this treatment to the case of anisotropic impurities - instead of "sphere-in-the-hole" we have to consider "ellipsoid-inthe-hole". In this case, there would be two factors determining the interaction between such centers: one is the elastic anisotropy of the host lattice, and another, specific for the JT case, is the dependence of the interaction on a relative orientation of the corresponding orbitals. In general, one has to consider both factors, but in the weakly anisotropic crystals it is often the second factor, which plays the dominant role, whereas the first one may differ from system to system.

\section{THE MODEL AND RELEVANT INTERACTIONS}

We consider below the situation with doubly degenerate $e_{g}$ orbitals, with one electron on them (e.g., ions $\mathrm{Mn}^{3+}\left(t_{2 g}^{3} e_{g}^{1}\right)$, low-spin $\left.\mathrm{Ni}^{3+}\left(t_{2 g}^{6} e_{g}^{1}\right)\right)$ or with one $e_{g}$ hole, $\mathrm{Cu}^{2+}\left(t_{2 g}^{6} e_{g}^{3}\right)$. As the basis orbitals one can take $d_{z^{2}}=\left|3 z^{2}-r^{2}\right\rangle$ and $d_{x^{2}-y^{2}}=\left|x^{2}-y^{2}\right\rangle$. The electron occupation of these orbitals corresponds to a quadrupolar distribution of electron density; the elongated electron ellipsoid for the electron $z^{2}$ orbitals, and the flattened (compressed) ellipsoid for $x^{2}-y^{2}$ orbitals. One may expect also the corresponding displacement of the ligands (e. g., the distortion of $\mathrm{O}_{6}$ octahedra): local elongation for $z^{2}$ electron and $x^{2}-y^{2}$ hole orbitals, and local compression in the opposite case. These local distortions will lead to a strain field in a crystal, which, as discussed above, will provide the mechanism of coupling between such ions. The latter can finally lead to the formation of one or another type of superstructures. This is the main mechanism considered in this paper.

There are, in principle, several aspects in the electronlattice interactions. The strain-induced interaction mentioned above is the interaction via long-wavelength phonons. This elastic interaction depends on the type of "impurity" and also on the anisotropy of the crystal. For anisotropic impurities, like JT centers considered in this paper, this interaction depends not only on the relative direction between sites in a crystal, but also on the relative local distortions on each site. As a result, this interaction becomes very complicated [16,17]. In addition to long-range interactions mediated by the strain field in a crystal, there exists also the interaction mediated by the short-range, or optical, vibrations. The detailed form 
of these interactions was studied in [12], and below we shall take these interactions into account. It turns out that the general structure of both these contributions is rather similar: the interactions between the nearestneighbor sites along crystal axes [100], [010], and [001], which can be obtained from [16] and [12], are exactly the same, and the interactions of next nearest neighbors along the diagonals [110], [011], etc. have the same sign, although somewhat different ratios. Thus, there are two contributions, which we consider below, the contribution due to the long-wavelength phonons (strain interaction), and that due to the nearest neighbor coupling via shortwavelength or optical phonons.

Generally speaking, one has to include also the electronic terms describing the exchange contributions to orbital ordering [3] and also an electron hopping, which can lead to delocalization of electrons. We consider below only the insulating states with localized electrons; therefore, we ignore electron hopping here, although in principle the tendency to delocalization and formation of metallic states can compete with the states considered below.

There is one assumption that we make below. In general, an arbitrary combination of the basic orbitals $z^{2}$ and $x^{2}-y^{2}$ of the type

$$
|\theta\rangle=\cos \theta / 2\left|z^{2}\right\rangle+\sin \theta / 2\left|x^{2}-y^{2}\right\rangle
$$

is allowed, corresponding both to local elongation and contraction of $\mathrm{O}_{6}$ octahedra or to any combination of these deformations (which would give both prolate and oblate ellipsoids or even biaxial ones). Experimentally it is well established, however, that only the local elongations are realized in practice: out of hundreds known Jahn-Teller compounds there are virtually none with compressed octahedra (for localized electrons) [3]. There are also physical reasons for that, related to the lattice anharmonicity and higher-order interactions [18,19]. In accordance to that, we will consider below only the situation with locally elongated "impurities", but the axes of local elongations in cubic crystals may be directed along $x, y$, or $z$ axes ([100], [010], or [001]), i.e. angles $\theta$ may be $0, \pm 2 \pi / 3$ for the one-electron orbitals as in $\mathrm{Mn}^{3+}$, and $\theta=\pi, \pm \pi / 3$ for the one-hole orbitals at $\mathrm{Cu}^{2+}$. Thus, we consider the case of elongated impurities (ellipsoids) interacting with one another via short-wavelength and long-wavelength phonons. The corresponding situations are illustrated in Figs. 1a and 1b for the neighbors along cubic axes $x, y$, and $z$, and in Figs. $1 \mathrm{c}$ and $1 \mathrm{~d}$ - for diagonal neighbors. It is qualitatively clear from these figures that the situation of Fig. 1a ( $z^{2}$ orbitals at nearest-neighbor pair along $z$ axis) would correspond to the repulsion (intermediate oxygen O1 feels "conflicting" forces), whereas that of Fig. 1b would give an attraction. This is indeed confirmed by the actual calculations [12, where the constants of these two and of two other possible situations were determined, see 1) -4) in Table 1 (this is actually the interaction via short-wavelength, or optical phonons).

The situation is somewhat more complicated for diagonal pairs like those in Figs. 1c and 1d. Here, it is the noncentral forces, which determine the resulting interactions. Still it is qualitatively clear that the interaction of oxygens O2 and O3 in Figs. 1c and 1d would lead to an effective repulsion in the first case and to an attraction in the second one (note that actually, in contrast to our figure, oxygen ions are the biggest ones, so that $\mathrm{O} 2$ and O3 "hard spheres" touch one another, which explain our conclusion). The coupling constants corresponding to Figs. 1c and 1d and for two other "diagonal" configurations (cases 5) - 8) in Table 1) we also take from [12].

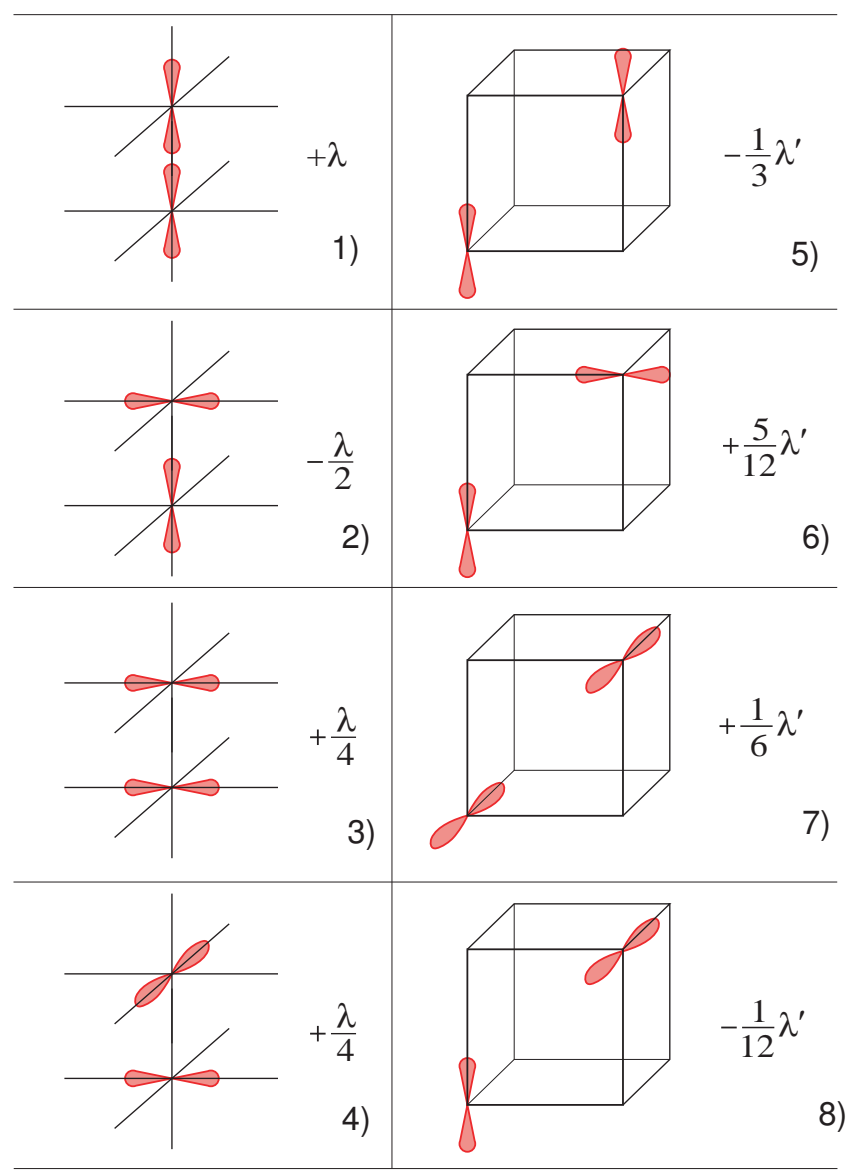

TABLE I. The interaction between different orbitals (local distortions) for nearest and next nearest neighbors. These interactions are expressed through two independent constants, $\lambda$ and $\lambda^{\prime}$, characterizing central (cases 1) -4)) and noncentral (cases 5) -8)) forces. The form and ratios of these interactions are taken from [12]; the long-range elastic interactions (3) give essentially the same expressions, see text. Here the electron orbitals are shown, in which case the local distortions have the same form (elongation along the electron cloud). For the one-hole case one should draw the orthogonal hole orbitals (e.g. in the case 1 ) - the hole orbitals $x^{2}-y^{2}$ ). 
Thus, the resulting nearest-neighbour interactions 1) - 8) are expressed through two parameters, $\lambda$ and $\lambda^{\prime}$, corresponding to the central and noncentral forces, respectively. Note also that these interactions take into account both the lattice structure (lattice anisotropy) and the anisotropic character of orbital occupation and of the on-site distortions.

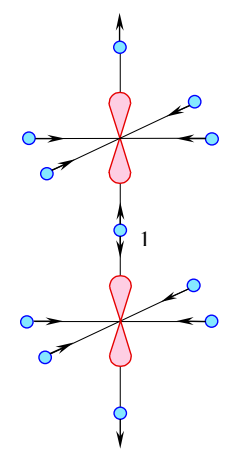

(a)

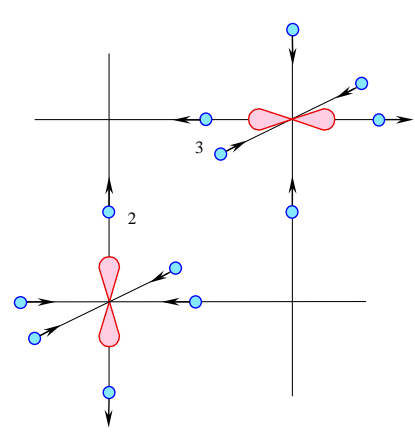

(c)

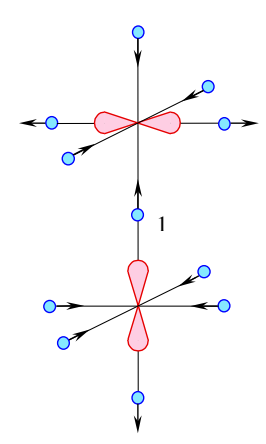

(b)

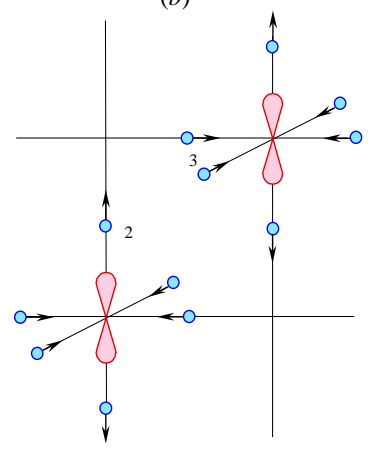

(d)
FIG. 1. Interactions between two Jahn-Teller ions with different occupied orbitals: (a) and (b) - pair along $z$ axis; (c) and $(\mathrm{d})$ - pair along the face diagonal of the perovskite unit cell. Displacements of oxygen ions are indicated by arrows.

The second contribution, due to interaction via the long-wavelength phonons for the case of impurities in the isotropic medium (i.e., for zero value of parameter $d$ defined by (2)) can be obtained from the general expression presented in [16]; it has the form

$$
\begin{aligned}
V= & \frac{\left(c_{11}+c_{44}\right)}{8 \pi\left(c_{11}+2 c_{44}\right) R^{3}}\left\{5 \sigma_{z z}^{(1)} \sigma_{z z}^{(2)}+\right. \\
& \left.+2\left(\sigma_{x x}^{(1)} \sigma_{x x}^{(2)}+\sigma_{y y}^{(1)} \sigma_{y y}^{(2)}\right)\right\}+ \\
& +\frac{1}{4 \pi R^{3}}\left(2 \sigma_{z z}^{(1)} \sigma_{z z}^{(2)}-\sigma_{x x}^{(1)} \sigma_{x x}^{(2)}-\sigma_{y y}^{(1)} \sigma_{y y}^{(2)}\right)
\end{aligned}
$$

where $c_{11}$ and $c_{44}$ are elastic moduli, and $\sigma_{\alpha \alpha}$ is a stress tensor such that e.g. for the center with the occupied orbital $3 z^{2}-r^{2}$ we have $\sigma_{z z}=1, \sigma_{x x}=\sigma_{y y}=-1 / 2($ and corresponding expressions for $z \rightarrow x, y)$.

The calculations show that the signs and the ratios of the interaction constants for the nearest neighbours (cases 1) - 4) in Table 1) obtained from (3), exactly coincide with those via short-wavelength phonons presented in Table 1. For the "diagonal" interactions (cases 4) - 8) in the Table 1) the signs of the interactions obtained from (3) are the same as those given in the Table 1, although the ratios of these constants differ somewhat. This is probably due to the assumption of the elastic isotropy made in $(3)$ (constant $d(2)$ is put $=0$ ), which is apparently less satisfactory for the noncentral forces.

For $d \neq 0$, the corresponding expression becomes much more complicated (see [16] for details). Often, for analysis of elastic interactions of impurities, further simplifications are introduced, namely the longitudinal and transverse sound velocities are taken to be equal. This leads to a rather simple explicit expression for the interaction between Jahn-Teller ions with different occupied orbitals, situated at arbitrary positions with respect to crystal axes [17]. For some problems (e.g. for the analysis of ESR spectra), such simplifications work reasonably well. However, in our case such approximation is definitely insufficient, leading to unphysical degeneracy in energies of different orbital configurations. At the same time, formula (3), as it was already mentioned, qualitatively correctly reproduces the main features of pair interactions even for nearest and diagonal neighbours in the cubic crystal.

Below, in calculating the energies of different ordered states, we would take into account only two types of interactions between the nearest neighbouring JT ions: those along cubic axes $x, y, z$, and along face diagonals. For this purpose, we will use the interaction constants presented in Table 1 [12], because they take into account both the anisotropy of JT ions and the elastic anisotropy of the lattice.

As we can see from Table 1, the interactions are determined by two independent constants: the first one, $\lambda$, corresponds to the directions along the cube axes (cases 1) -4)) and is determined by central forces between ions, and another one, $\lambda^{\prime}$ - along diagonals of the elementary plaquettes - is caused by the noncentral forces [12]. For the elastic interactions, these constants are determined by different elastic moduli of the crystal: by the bulk and shear moduli, respectively.

In principle, one should include also longer-range interactions, but their due account would require rather extensive numerical calculations, which we postpone for future publications. Still, we will show below that even using only the nearest neighbor interactions, we can successfully describe many superstructures observed e.g. in manganites at different doping levels, and can analyze such questions as the relative stability of single $v s$ paired stripes, compare the energy of different types of orbital 
domain walls, etc. One may hope that the longer-range interactions, which are still much weaker than those taken into account, would not modify our main conclusions.

\section{IV. $\mathrm{LaMnO}_{3}, \mathrm{UNDOPED} \mathrm{LAYERED}$ MANGANITES AND OTHER SIMILAR MATERIALS}

First, we consider the simplest case of undoped oxides, which may be relevant for such systems as undoped perovskite $\mathrm{LaMnO}_{3}$ or $\mathrm{KCuF}_{3}$, layered materials like $\mathrm{K}_{2} \mathrm{CuF}_{4}$, etc. These systems contain ions $\mathrm{Mn}^{3+}\left(t_{2 g}^{3} e_{g}^{1}\right)$ or $\mathrm{Cu}^{2+}\left(t_{2 g}^{6} e_{g}^{3}\right)$ at each lattice site, forming a simple cubic lattice for $\mathrm{LaMnO}_{3}$ and square perovskite-like lattice in layered systems.

IVa. First, we consider the 2D square lattice relevant for layered systems. One immediately sees that if the $\left|3 z^{2}-r^{2}\right\rangle$ and $\left|x^{2}-y^{2}\right\rangle$ orbitals are exactly degenerate, then with the interactions presented in Table 1 one would get the structure shown in Fig. 2. This structure is stabilized first of all by the attraction $(-\lambda / 2)$ of orthogonal orbitals (deformations) $x^{2}$ and $y^{2}$ along $x$ and $y$ directions. The diagonal coupling is that of parallel orbitals (case 5) in Table 1), i.e. it is also attractive $\left(-\lambda^{\prime} / 3\right)$ and gives extra stabilization of this configuration. Its energy (per site) is

$$
E=2(-\lambda / 2)+2\left(-\lambda^{\prime} / 3\right)=-\lambda-2 \lambda^{\prime} / 3 .
$$

One can easily see that all the other possible orderings of these local distortions (locally elongated octahedra) give higher energy. The structure described above coincides with the ordering observed in the layered cuprate $\mathrm{K}_{2} \mathrm{CuF}_{4}$ 20] (which was predicted earlier on different grounds 21]). The only difference is that for $\mathrm{Cu}^{2+}$ such a packing of local distortions corresponds not to an alternation of electron orbitals $x^{2}$ and $y^{2}$, but to the corresponding pattern formed by the hole orbitals $\left(y^{2}-z^{2}\right.$ and $\left.x^{2}-z^{2}\right)$. The same ordering is also observed in layered chromites $\mathrm{Rb}_{2} \mathrm{CrCl}_{4}$, see [3,22].

Due to a layered structure of corresponding systems there may exist in them an initial splitting of the $z^{2}$ and $\left(x^{2}-y^{2}\right)$-orbitals corresponding to local elongation along $c$-direction, and if this splitting is large enough, it can give the ferro-orbital ordering such as the $x^{2}-y^{2}$ ordering in $\mathrm{La}_{2} \mathrm{CuO}_{4}$. For layered manganites (singlelayer $\mathrm{La}_{1-x} \mathrm{Sr}_{1+x} \mathrm{MnO}_{4}$, bilayer $\mathrm{La}_{2-2 x} \mathrm{Sr}_{1+2 x} \mathrm{Mn}_{2} \mathrm{O}_{7}$ ), we would expect in this case the $z^{2}$ occupation at each cite. This is apparently the situation in the undoped $(x$ $=0$ ) layered manganites [23.

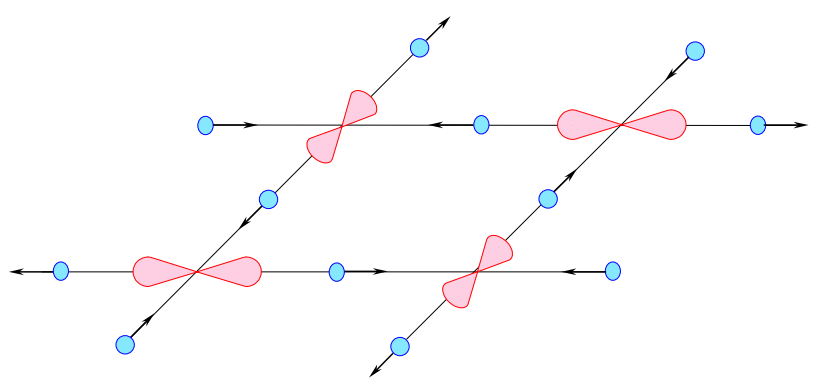

FIG. 2. Possible packing of local distortions and orbital structure (occupied electron orbitals) for undoped layered systems. Oxygen shifts are shown by arrows

IVb. Let us now consider the case of a cubic perovskite $\mathrm{LaMnO}_{3}$ (we ignore at first the tilting of $\mathrm{MnO}_{6}$ octahedra leading to the orthorhombicity). One immediately sees that the same factors, which acted in layered systems, would stabilize the superstructure of Fig. 2 in the basal planes of $\mathrm{LaMnO}_{3}$. The complication arises, when one considers the ordering pattern in the third $(z$ axis) direction. One can have two types of arrangement of such planes. They can form either the in-phase structure (Fig. 3a), or the out-of-phase structure (Fig. 3b). These are usually referred to as the $d$-type and $a$-type structures, respectively. With the interactions of Table 1, their energies turn out to be the same:

$$
\begin{aligned}
E_{3 a} & =2\left(-\frac{\lambda}{2}\right)+\frac{\lambda}{4}+2\left(-\frac{\lambda^{\prime}}{3}\right)+4\left(-\frac{\lambda^{\prime}}{12}\right) \\
& =-\frac{3}{4} \lambda-\lambda^{\prime} \\
E_{3 b}=2 & \left(-\frac{\lambda}{2}\right)+\frac{\lambda}{4}+2\left(-\frac{\lambda^{\prime}}{3}\right)+2 \frac{\lambda^{\prime}}{6}+2\left(-\frac{\lambda^{\prime}}{3}\right) \\
= & -\frac{3}{4} \lambda-\lambda^{\prime}
\end{aligned}
$$

Experimentally, both types of ordering are observed in perovskites: ordering of the $d$-type (Fig. 3a) is found e.g. in $\mathrm{LaVO}_{3}$, and the $a$-type structure - in $\mathrm{YTiO}_{3}$. In $\mathrm{KCuF}_{3}$ both types of ordering may arise, and stacking faults are formed very easily. For $\mathrm{LaMnO}_{3}$, only the ordering of the $d$-type (Fig. 3a) is observed. Apparently, there are some other factors, not included on our model, which determine the ordering in third direction. 


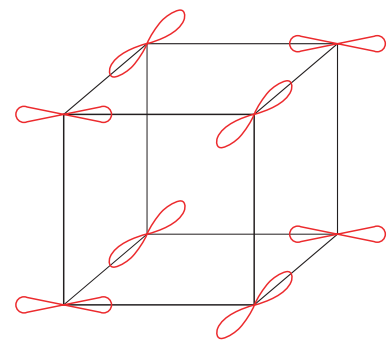

(a)

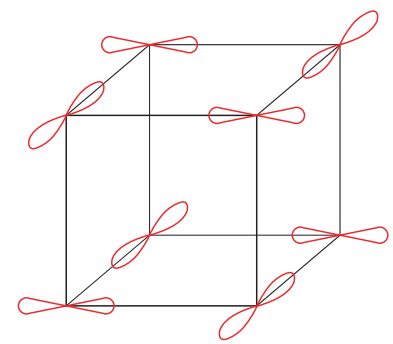

(b)
FIG. 3. Two different kinds of orbital ordering in cubic manganites: (a) in-phase and (b) out-of-phase.

One such factor may be an interplay of the Jahn-Teller distortions with the $\mathrm{GdFeO}_{3}$-type distortion caused by the tilting of the $\mathrm{MeO}_{3}$ octahedra 25] - one may show that for strong enough tilting this interplay stabilizes the $d$-type ordering of Fig. 3a. In any case, the main motive - packing of the distortions shown in Fig. 2 - is the first and the most important ingredient in the orbital superstructures observed in many perovskites with JT ions, and this directly follows from our mechanism.

Thus, we see that the electron-lattice coupling quite naturally gives the correct lattice and orbital superstructure of undoped manganites (see also [12,24])

\section{LOW-DOPED MANGANITES}

There are several interesting systems, which show rather puzzling properties at low doping level. Thus, $\mathrm{La}_{1-x} \mathrm{Sr}_{x} \mathrm{MnO}_{3}$ has an insulating ferromagnetic state at about $x=1 / 8(\sim 0.1 \leq x \leq 0.14)[26,27$. Such a state appearing in oxides is in general a rare occasion: most of the insulating systems are antiferromagnetic, and ferromagnetism usually coexists with (and is explained by) the metallicity (double exchange). Probably, the only way to stabilize a ferromagnetic insulating state is by forming a special orbital ordering [ [ 1 , so as to give ferromagnetic exchange according to the aforementioned GoodenoughKanamori-Anderson rules. The superstructure was indeed observed in $\mathrm{La}_{1-x} \mathrm{Sr}_{x} \mathrm{MnO}_{3}$ in this region [28], and it was interpreted in 28] as predominantly the charge ordering - ordering of holes ( $\mathrm{Mn}^{4+}$ ions) of more or less $b c c$ type in the $\mathrm{Mn}^{3+}$ matrix. The remaining $\mathrm{Mn}^{3+}$ ions, of course, should have certain orbital occupation in an insulator, which could in principle finally lead to ferromagnetism.

This problem was considered theoretically in [29, where it was shown that if one assumes the charge ordering pattern of Yamada et al. [28], one would indeed obtain certain orbital ordering, which can be treated as an ordering of orbital polarons 30.31 leading to a ferromagnetic spin ordering. These orbital polarons are the objects, in which the central small-size $\mathrm{Mn}^{4+}$ ion is surrounded by larger $\mathrm{Mn}^{3+}$ ions, with the lobes of their orbitals directed toward the central $\mathrm{Mn}^{4+}$. The elastic forces are definitely rather important in forming such objects and in their possible ordering (see also [32]). However, the Hartree-Fock calculations carried out in 29] have shown that the better structure with the lower energy is the one, in which the $x y$ planes alternate as follows: one plane is a pure $\mathrm{Mn}^{3+}$ plane with the superstructure of Fig. 2; the next plane contains all the holes $\left(\mathrm{Mn}^{4+}\right.$ ions) forming "vertical" stripes (stripes along $x$ or $y$ directions), see Fig. 4. Such a state turned out to be also ferromagnetic; this structure is consistent with the lattice symmetry observed in [28].

Although the numerical calculations of 29] were carried out using different starting point, one can argue that it is the special stability of the orbital ordering of Fig. 2 that helps to stabilize (or is even a driving force of) such a superstructure in low-doped manganites, with segregation of pure undoped $\mathrm{Mn}^{3+}$ planes, all the holes being in other planes. If it is true, one can expect similar phenomena also in other systems, e.g. in low-doped $\operatorname{Pr}_{1-x} \mathrm{Ca}_{x} \mathrm{MnO}_{3}$, where the ferromagnetic insulating phase is also known to exist at $0.1 \leq x \leq 0.3$ [33, 39]. It is possible in principle that the structure of this system, e.g. close to $x=1 / 4$, is also formed by the ordered array of orbital polarons [31. But the better alternative may be again the segregation of holes in every second plane, undoped planes being indeed like those in Fig. 2, and doped planes (which in this case would have the effective doping $x_{\text {eff }}=n_{\text {holes }}=0.5$ ) having either stripe-like structure or even the superstructure of the CE type, characteristic of $x=0.5$ (see below).

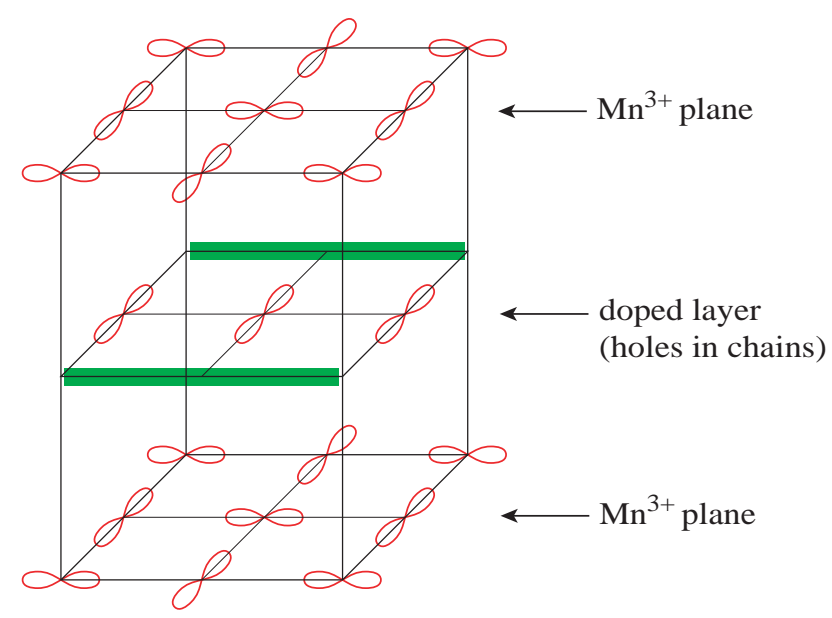

FIG. 4. A possible orbital ordering at $n=1 / 8$.

The detailed type of charge and orbital ordering in $\mathrm{Pr}_{1-x} \mathrm{Ca}_{x} \mathrm{MnO}_{3}$ in the vicinity of $x=1 / 4$ is not known yet, although the first measurements by the anomalous X-ray scattering [35] have shown that in this system there indeed exists a superstructure, which can be consistent 
with that in Fig. 4 and/or with the CE type superstructure of Fig. 5a. It would be very interesting to check this possibility experimentally in more detail.

\section{HALF-DOPED SYSTEMS: CHARGE AND ORBITAL ORDERING}

\section{VIa. Ground state}

In most of half-doped manganites $R_{1-x} \mathrm{Me}_{x} \mathrm{MnO}_{3}$, $x=0.5(R=\mathrm{La}, \mathrm{Pr}, \ldots)$, there exist at low temperatures the charge and orbital ordering accompanied by the antiferromagnetic ordering of the CE type [38,39], the latter usually appearing at still lower temperatures. Nowadays, one often uses the term "CE ordering" also to denote the corresponding charge and orbital structures. This ordering is illustrated in Fig. 5a. It consists of the checkerboard arrangement of $\mathrm{Mn}^{3+}$ and $\mathrm{Mn}^{4+}$ ions with the corresponding orbital ordering at $\mathrm{Mn}^{3+}$ sites. Of course, one should not take notations " $3+$ " and " $4+$ " too literally: actual degree of charge disproportionalization can be much less, e.g., $\sim(3.5 \pm 0.2)$ [37]. This is the structure in the basal plane; the stacking of these planes in the third direction is in phase, so that $3+$ and $4+$ sites, as well as the corresponding orbitals, are on top of one another.

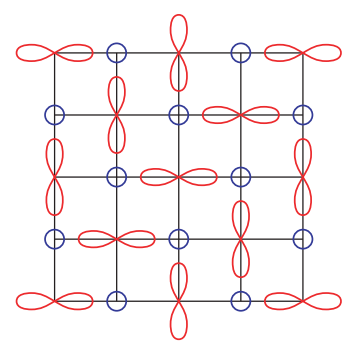

(a)

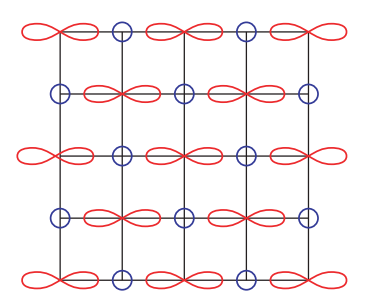

(b)
FIG. 5. Charge and orbital orderings in half-doped manganites: (a) actual ordering (the so called CE structure); (b) alternative orbital ordering with all occupied orbitals at $\mathrm{Mn}^{3+}$ of the same type $\left(x^{2}\right.$ orbitals). Empty circles denote $\mathrm{Mn}^{4+}$ ions, the electron density distribution for the corresponding orbitals is shown for the $\mathrm{Mn}^{3+}$ sites.

There are two questions related to this superstructure. The first one concerns the type of charge ordering. It is quite natural to expect the checkerboard arrangement of $\mathrm{Mn}^{3+}$ and $\mathrm{Mn}^{4+}$ ions, or of extra electrons: such ordering is actually analogous to a Wigner crystal, i.e. it is favored by the Coulomb forces. These considerations, however, are definitely not enough, because in this model one would expect charge (valence) alternation also in the third direction, which is not the case. The solution of this problem may again be connected with the elastic interactions: it can be shown [14,40] that they typically favor the inclusion of one phase (e.g. $\left.\mathrm{Mn}^{4+}\right)$ in another $\left(\mathrm{Mn}^{3+}\right)$ in the form of infinitely thin slabs - sheets, oriented in a crystal in a particular way, so as to minimize total strain energy. These may be just $\mathrm{Mn}^{4+}$ sheets observed in the CE structure.

Another question concerns the type of orbitals occupied at $\mathrm{Mn}^{3+}$ sites. If we assume the checkerboard charge ordering, we may have different types of occupied orbitals, e.g., one can in principle get the ordering shown in Fig. 5b with all orbitals of the same type (here, $x^{2}$ orbitals) instead of alternation of $x^{2}$ and $y^{2}$ diagonal rows ("stripes") of Fig. 5a.

The type of orbital ordering in the checkerboard charge structure can be again analyzed using the interactions listed in Table 1. Here we also include the interaction of two types of nearest neighbors: the first neighbors in the $\mathrm{Mn}^{3+}$ sublattice are here the diagonal interactions $5)-8$ ) of Table 1 , and the second neighbors are those along $x$ and $y$ directions in Fig. 5. The only difference is that these interactions are now at twice the distance of the nearest neighbors $\mathrm{Mn}^{3+}$ and $\mathrm{Mn}^{4+}$ in these directions, so that according to the general nature of elastic interactions, which decay as $1 / R^{3}$, these couplings with parameter $\lambda$ (cases 1) - 4) in Table 1) should be now multiplied by factor $1 / 2^{3}=1 / 8$.

From Table 1 one sees that the diagonal interactions are attractive for the same orbitals, e.g., $x^{2}$ and $x^{2}$ (case 5 ) in Table 1), but repulsive for $x^{2}$ and $y^{2}$ (case 6)). From this point view, one should rather expect that the orbital structure in this case would correspond to Fig. 5b, and not to Fig. 5a. However, the interactions along $x$ and $y$ directions tend to stabilize the structure of Fig. 5a. One can easily calculate the energies of these two competing states (per site):

$$
E_{5 a}=\left(\frac{-\lambda^{\prime}}{3}\right)+\left(\frac{5 \lambda^{\prime}}{12}\right)+2 \cdot \frac{1}{8}\left(\frac{-\lambda}{2}\right)=\frac{\lambda^{\prime}}{12}-\frac{\lambda}{8}
$$

Here, the first two terms are the diagonal interactions with the first neighbouring $\mathrm{Mn}^{3+}$ ions and the last term is the interaction along $x$ and $y$ directions (at a distance of two lattice parameters).

Similarly, the energy of the state presented in Fig. 5b is

$$
E_{5 b}=2\left(\frac{-\lambda^{\prime}}{3}\right)+\frac{\lambda}{8}+\frac{1}{8}\left(\frac{-\lambda}{4}\right)=-\frac{2 \lambda^{\prime}}{3}+\frac{5 \lambda}{32}
$$

Thus, if the central force interaction $\lambda$ is strong enough,

$$
\lambda^{\prime} / \lambda<3 / 8=0.375
$$

the CE structure of Fig. 5a would be stable; for the opposite inequality one would get the structure of Fig. 5b. Since actually the CE structure is observed in experiment, one should conclude that inequality (9) is fulfilled in manganites (within the approximations made). One 
can also show that all other feasible types of orbital orderings have higher energy.

One has to stress that the assumption of the checkerboard charge ordering is crucial for this conclusion; if we would not make this assumption and use only the JTinduced interactions of Table 1, one should rather get in the model under study the phase separation: all electrons $\left(\mathrm{Mn}^{3+}\right.$ ions) would form a dense cluster with the superstructure of Fig. 2. This would minimize the interaction energy of the JT-distorted sites. Thus one has to add some extra factors to this model, e.g., the Coulomb interaction preventing such phase separation. However, assuming the checkerboard charge ordering, we can explain the concomitant orbital ordering of Fig. 5a in our model. (Nevertheless, the possibility of phase separation cannot be completely discarded even in this case - and all the more so at $x \neq 0.5$ 41].)

VIb. Orbital domains

It is also of interest, and of practical importance, to study in our model the "cost" of creation of defects, notably orbital domain walls. As argued in [34] and especially in [36], such domain walls are easily formed in $\mathrm{CE}$ states, e.g. in $\mathrm{La}_{0.5} \mathrm{Ca}_{0.5} \mathrm{MnO}_{3}$ and in $\mathrm{Pr}_{1-x} \mathrm{Ca}_{x} \mathrm{MnO}_{3}$.

One can easily calculate in our model the energy of such defects. The simplest one is the local defect - rotation of the orbital on one site, e.g. from $x^{2}$ to $y^{2}$. With our interaction constants it would cost us $\Delta E=9 / 16 \lambda$ (diagonal interactions remain the same for the real $\mathrm{CE}$ structure corresponding to Fig. 5a). More interesting is the situation with the domain walls. There are four types of them, classified and drawn in Figs. 3b-3e of [36]. Domains separated by domain walls $3 \mathrm{~b}$ and $3 \mathrm{c}$ of [36] are those with the same propagation vector, i.e. the same orientation of orbital stripes, and $3 \mathrm{~d}$ and $3 \mathrm{e}$ are domain walls between two domains with the propagation vectors rotated by 90 degrees (orbital twins). Straightforward calculations show that the energies of domain walls (per unit length, i.e. per $\mathrm{Mn}^{4+}$-site at the center of domain wall) are

$$
\begin{gathered}
\Delta E_{3 b}=-\frac{3}{4} \lambda^{\prime}+\frac{9}{32} \lambda \\
\Delta E_{3 c}=\frac{3}{4} \lambda^{\prime}+\frac{9}{32} \lambda \\
\Delta E_{3 d}=\Delta E_{3 e}=\frac{\Delta_{3 b}}{2}=\frac{1}{2}\left[-\frac{3}{4} \lambda^{\prime}+\frac{9}{32} \lambda\right]
\end{gathered}
$$

As we see from (10), the domain wall $3 \mathrm{c}$ is the most "expensive" one, it always has positive creation energy. The situation with other three types of domain walls, $3 \mathrm{~b}, 3 \mathrm{~d}$, and $3 \mathrm{e}$ in the notation of [36], is different: they cost us some energy for small $\left(\lambda^{\prime} / \lambda\right)$, but they can be created spontaneously $(\Delta E<0)$ if diagonal coupling $\lambda^{\prime}$ is sufficiently strong. As we see from (10), the critical value for this coincides with the critical value $\left(\lambda^{\prime} / \lambda\right)=3 / 8(9)$ obtained above, which confirms the consistency of our treatment.

An important conclusion is that, at least in our model, in the range of existence of the CE structure, $\left(\lambda^{\prime} / \lambda\right)<$ $\left(\lambda^{\prime} / \lambda\right)_{c}=3 / 8$, the domain walls of the type $3 \mathrm{~d}$ and $3 \mathrm{e}$ are easiest to form, and they have the same creation energy. Thus, it will be presumably orbital twins $3 \mathrm{~d}$ and 3e which will appear in real systems. The driving force for their formation is apparently the same as in ordinary martensitic transitions [40], i.e. they are elastic domains caused by strain in crystals (this is suggested also by A. Millis, as cited in [36]). different from what was usually assumed: instead of the

\section{STRIPES AND BISTRIPES IN OVERDOPED MANGANITES}

Finally, we turn to the most controversial problem - that of the type and the origin of superstructures in overdoped manganites, e.g. stripes and bistripes in $\mathrm{La}_{1-x} \mathrm{Ca}_{x} \mathrm{MnO}_{3}$ at $x=2 / 3 ; 3 / 4$ [6] 8]. First of all, there exists an experimental controversy: different experiments give for the same system and even for the same samples different results. Thus, for $x=2 / 3$ the high-resolution electron microscopy gave the results taken as a signature of paired stripes or bistripes [8], whereas the neutron scattering results were interpreted in terms of single stripes (called "Wigner crystal" in [6]).

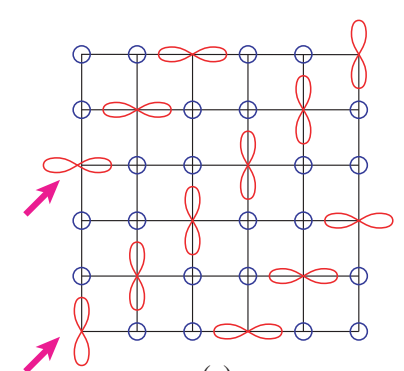

(a)

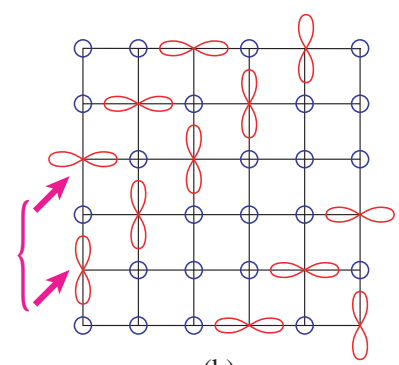

(b)

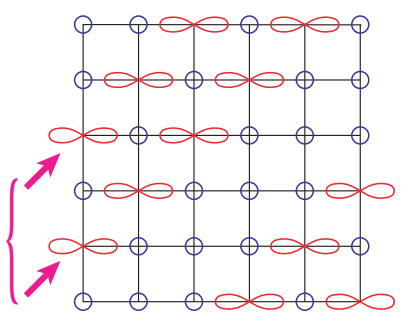

(c)

FIG. 6. Possible stripe structures in the basal plane of $\mathrm{La}_{1-x} \mathrm{Ca}_{x} \mathrm{MnO}_{3}$ at $x=2 / 3$ : (a) single stripes; (b) bistripes with different orbitals; (c) bistripes with similar orbitals. 
These two situations, with the corresponding orbital ordering, are illustrated in Fig. 6a, b for the case $x=2 / 3$. The orbitals at each stripe are parallel, e.g. one stripe is formed by $x^{2}$ orbitals and another stripe by $y^{2}$ orbitals. In the paired stripe picture (Fig. 6b), these two diagonal stripes, with $x^{2}$ and $y^{2}$ orbitals, come close together being separated by one $\mathrm{Mn}^{4+}$ diagonal row.

Again, we have here several questions: why stripes here are better than any other type of charge ordering? (e.g., for $x=3 / 4$, one can arrange the real "Wigner crystal" structure with the $\mathrm{Mn}^{3+}$ sites forming a face-centered 2D lattice); and why do stripes have a particular orbital structure? And the third, most burning question is which state, single or paired stripes, is better?

One can try to approach these problems using our model with the interactions of Table 1 , adding to it a restriction forbidding the formation of dense $\mathrm{Mn}^{3+}$ clusters. Similar to Section VI, we calculate now the energies of the single stripes of Fig. 6a, paired stripes of Fig. 6b, and also the possible competing type of bistripes shown in Fig. 6c, with all orbitals of the same type, e.g. $x^{2}$. Using the interaction parameters of Table 1 and keeping again the interaction of the first (diagonal) and of the second "direct" neighbours at a distance of two lattice constants in $x$ and $y$ directions, we obtain:

$$
\begin{gathered}
E_{6 a}=-\frac{\lambda^{\prime}}{3} \\
E_{6 b}=-\frac{\lambda^{\prime}}{3}+\frac{1}{2} \frac{5 \lambda^{\prime}}{12}+\frac{1}{8}\left(-\frac{\lambda}{2}\right)=-\frac{\lambda^{\prime}}{8}-\frac{\lambda}{16}, \\
E_{6 c}=\frac{3}{2}\left(-\frac{\lambda^{\prime}}{3}\right)+\frac{1}{2}\left(\frac{\lambda}{8}+\frac{1}{8} \frac{\lambda}{4}\right)=-\frac{\lambda^{\prime}}{2}+\frac{5 \lambda}{64} .
\end{gathered}
$$

Expression (11) is quite clear. In (12), the first term describes the diagonal interaction along the stripe, the second - diagonal interactions between stripes in the pair (each such bond belongs to two sites, therefore we have factor $1 / 2$ in it), and the third term is the next-nearestneighbour interaction of $x^{2}$ orbital of one stripe with the $y^{2}$ orbitals of its "mate". Similarly, one can understand the meaning of different terms in (13).

Comparing the energies of these three states, one obtains that there exist two critical values of parameter $\nu=\lambda^{\prime} / \lambda$

$$
\begin{gathered}
\nu_{c r}^{(a b)}=3 / 10=0.3, \\
\nu_{c r}^{(a c)}=15 / 32 \simeq 0.47 .
\end{gathered}
$$

For $\lambda^{\prime} / \lambda<\nu_{c r}^{(a b)}=0.3$, the paired stripes of Fig. $6 \mathrm{~b}$ are stable. For $0.3=\nu_{c r}^{(a b)}<\lambda^{\prime} / \lambda<\nu_{c r}^{(a c)}=0.47$, the single-stripe phase of Fig. 6a would have the lowest energy among the three states, which we compare. And finally, for $\lambda^{\prime} / \lambda<\nu_{c r}^{(a c)}=0.47$, the paired stripes of Fig. $6 \mathrm{c}$ would have the lowest energy. We again discard here the possibility of phase separation, where all electrons are assembled in the bulk CE-like phase of Fig. 5a, which can arise at small $\lambda^{\prime} / \lambda$, and into the phase of the type shown in Fig. 5b for large $\lambda^{\prime} / \lambda$, assuming that such phase separation into electron-enriched and electron-depleted regions would be prevented, e.g. by the long-range Coulomb interaction.

Comparing these results with those of Section VIa (half-doped systems), we can see that for realistic values of $\lambda^{\prime} / \lambda<0.375$ (9), which are needed to stabilize the experimentally observed CE phase, we may have either the paired stripes [8] (Fig. 6b), or single stripe phase of Fig. 6a, see phase diagram in Fig. 7. Unfortunately, within this treatment we cannot make a unique choice between these two possibilities. Nevertheless, we can say that at least the sometimes used arguments [8], which ascribe the pairing of stripes to strain interactions, are not very convincing: we see that the same strain interactions for different ranges of parameters may rather favour single stripes. Apparently, a more detailed treatment, including also long-range interactions, is needed to answer theoretically the question, which state, single or paired stripes, would be preferred for real systems (although it is, of course, primarily an experimental problem).

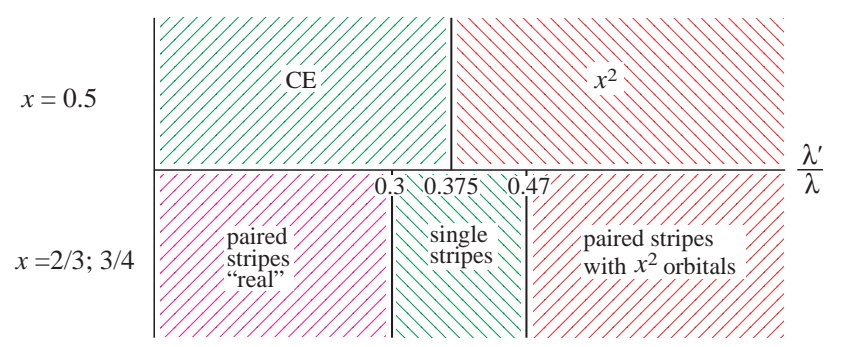

FIG. 7. Regions of stability of different phases for $x=0.5$ (upper half-plane) and for overdoped manganites $(x=2 / 3$; $3 / 4$, lower half-plane). Note that we do not consider fully phase-separated states.

As an indirect argument we may only use the results of the study of domain walls at the end of Section VI. As shown there, the energy cost of domain wall formation goes to zero at the critical point $\left(\lambda^{\prime} / \lambda\right)=0.375$ (9). As follows from the experimental data 36], orbital domains are formed very easily in half-doped manganites. It may be a signature that real systems are not far from this critical point. If true, this would mean that, according to the results (12) and (13) and to Fig. 7, single stripes may be preferable, although the energy of paired stripes may be not very far from it, so that they may become stable e.g. at the surface of the crystal. This could resolve the experimental controversy between the results of [6] and [8]. 


\section{MODELING OF ORBITAL ORDERING BY INTERACTING QUADRUPOLES}

As is well known [3], the orbital ordering is closely related to the ordering of electric quadrupoles: the asymmetric distribution of charge density at a transition metal ion with a particular occupation of one of degenerate orbitals corresponds to a quadrupolar charge distribution, and, moreover, the interaction of orbitals via lattice distortions is very similar to the conventional quadrupolequadrupole $(\mathrm{QQ})$ interaction. It is instructive to see whether one can model the elastic interactions listed in Table 1 by this QQ interaction. Of course, in real transition metal compounds this direct QQ interaction is much weaker than that via phonons (it may be relevant though for rare earths compounds, where one indeed often uses the terminology "quadrupolar ordering" instead of our "orbital ordering" and "cooperative Jahn-Teller effect"). The distance dependence is also different: the QQ interactions decay as $1 / R^{5}$, whereas the interactions via phonons - only as $1 / R^{3}$. Nevertheless, the operator structure of the corresponding terms in the Hamiltonian is very similar, and one may expect that at least the signs, and probably the ratios of corresponding coupling constants would be similar for both these mechanisms.

The general form of interaction between two quadrupoles $\mathrm{Q}_{\alpha \beta}$ and $\mathrm{Q}_{\gamma \delta}$, located in positions with radius vector $\mathbf{R}$ connecting them, can be obtained from the general treatment 42]:

$$
\begin{aligned}
U_{Q Q^{\prime}}= & \frac{5}{12 R^{5}}\left[7 Q_{\alpha \beta} Q_{\gamma \delta}^{\prime} n_{\alpha} n_{\beta} n_{\gamma} n_{\delta}-\right. \\
& \left.4 Q_{\alpha \beta} Q_{\alpha \gamma}^{\prime} n_{\beta} n_{\gamma}+\frac{2}{5} Q_{\alpha \beta} Q_{\alpha \beta}^{\prime}\right] d V
\end{aligned}
$$

where $n_{\alpha}$ etc. are directional cosines of radius-vector $\mathbf{R}$.

Using this general expression for axially symmetric quadrupoles $\left(Q_{z z}=Q, Q_{x x}=Q_{y y}=-Q / 2\right)$, one can easily calculate the quadrupolar interaction constants for the cases 1) - 8) of Table 1. They turn out to be

$$
\begin{array}{r}
V_{1)}=\Lambda ; V_{2)}=-\frac{\Lambda}{2} ; \quad V_{3)}=\frac{\Lambda}{8} ; \quad V_{4)}=\frac{3 \Lambda}{8} ; \\
V_{5)}=-\frac{1}{(\sqrt{2})^{5}} \frac{13 \Lambda}{40} ; \quad V_{6)}=\frac{1}{(\sqrt{2})^{5}} \frac{49 \Lambda}{96} ; \\
V_{7)}=\frac{1}{(\sqrt{2})^{5}} \frac{3 \Lambda}{8} ; \quad V_{8)}=-\frac{1}{(\sqrt{2})^{5}} \frac{3 \Lambda}{8},
\end{array}
$$

where $\Lambda=3 Q^{2} / 2 R^{5}$. In calculating the values of $V_{1)}-V_{8}$ ) in (17), we separated out the factor $1 /(\sqrt{2})^{5}$, since the distance for diagonal pairs is larger by a factor of $\sqrt{2}$ than that for the "direct" nearest neighbours.

One can see that indeed the signs of the QQ interactions in (17) coincide with those of elastic interactions (see Table 1); this gives an extra support to these values used in the main part of our paper. Moreover, even the ratios of the interaction constants are rather close, at least for cases 1) - 4). In principle, one can try to find out the type of the ordered ground state for the QQ case, using general expression (16) and taking into account the longer-range interactions and interactions for all possible directions. This task would require rather extensive numerical calculations (or some clever summation of the Madelung type); we do not do this here. One can only formulate some interesting problems, which should be addressed in this field.

(1) Would there be indeed a long-range ordered state in this system? We remind that for a similar problem with the dipole, not quadrupole, interactions it was proven 43 using the approach similar to that of Mermin-Wagner 44. that there is no long-range ordering for the dipole-dipole system in the case of 3D cubic lattice. Such possibility is not excluded also for the QQ interaction (for systems with quadrupoles at each lattice site).

(2) Another curious problem is what would be the situation with the mobile quadrupoles if their concentration is small (their number is less than the number of lattice sites). Such quadrupoles definitely attract each other at certain directions and for certain relative orientations of their main axes. Would they form structures like stripes, sheets, or some more complicated superstructures, and which in particular? Note that this possibility is rather similar to the one suggested in 14 for elastic interactions of spherical "impurities" in anisotropic media. Here, however, one considers the interaction of anisotropic centers in the isotropic space. Nevertheless, the outcome may be similar (actually the consideration of Section IV have much in common with this problem; but for the QQ case one can go beyond approximation of the nearest-neighbor interactions made above and consider the problem in its full complexity). Note also that similar question exists also for mobile dipoles.

These situations have not only an academic interest: one can imagine that similar superstructures of various kinds can arise due to these interactions, e.g. in the solutions of anisotropic molecules in certain solvents, etc.

The questions discussed in this Section have only indirect relation to the main problem considered in the present paper, but still we see much in common between these situations, at least in principle.

\section{CONCLUSIONS}

In conclusion, we considered in this paper the possibility of formation of different superstructures in insulating systems with Jahn-Teller ions like some cuprates, chromites, and especially manganites at different doping levels. We saw that even keeping only the interactions 
between a few nearest neighbors, we can successfully describe the formation of different orbital superstructures. In particular, using only the elastic interactions one can immediately obtain the observed ordering pattern of undoped manganites. By assuming the checkerboard charge ordering, we can easily explain by this mechanism the type of orbital ordering (CE structure) observed in halfdoped manganites $(x=0.5)$. Using the same physical arguments, we suggested that the superstructures observed in the low-doped manganites (ferromagnetic insulating phases at $x=0.1-0.3)$ involve the segregation of doped holes in consecutive planes, this structure being favored by the special stability of the orbital ordering of the "undoped $\mathrm{LaMnO}_{3}$-type" - the ordering shown in Fig. 2. For the overdoped manganites like $\mathrm{La}_{1-x} \mathrm{Ca}_{x} \mathrm{MnO}_{3}, x>0.5$, we argued that the stability of stripes or bistripes observed in this doping range (especially at $x=2 / 3 ; 3 / 4$ ) is also determined by the elastic forces, although we were not able to determine uniquely which of these alternatives, single or paired stripes, is more favorable. In our approach, the result depends on the ratio of two constants related to the noncentral and central forces respectively, which we do not know a priori. But in any case, the elastic forces largely determine the very tendency to the stripe formation in insulating overdoped manganites, as well as their orbital structure.

As mentioned in the Introduction, there exist also other possible mechanisms of orbital ordering, in particular, the superexchange interaction [3,13, Typically both these mechanisms, the exchange interaction and the interaction via lattice, lead to the same orbital structures in cases when we have Jahn-Teller ions at each site. It is quite difficult to find the cases, in which the outcomes of these two models would be qualitatively different 45]. In some sense, it is gratifying that very simple considerations presented above immediately give the correct orbital structures for the cases like $\mathrm{LaMnO}_{3}$ or $\mathrm{K}_{2} \mathrm{CuF}_{4}$, whereas rather hard work is required to get these structures with the electronic (exchange) mechanism. Thus, apparently both these mechanisms are operational in real systems, and the relative importance of each is still an open problem, at least for the cases of dense systems (see also 46] in this context).

But when we turn to the dilute systems, like doped manganites, especially in the overdoped regime $x>0.5$, then apparently the elastic interactions play the most important role, first of all providing the essential mechanism of stripe formation, and also determining their orbital structure: one can expect that the exchange interactions, being essentially short-range, are less efficient in these cases. It is mainly in these systems, where we believe that our approach may be most fruitful.

\section{ACKNOWLEDGMENTS}

The work was supported by INTAS (grants 97-0963 and 97-11954), the Russian Foundation for Basic Research (grant 00-15-96570), the Netherlands Foundation for the Fundamental Research of Matter (FOM), and the Netherlands Organization for the Advancement of Pure Research (NWO).

[1] J. B. Goodenough, Magnetism and the Chemical Bond (Interscience, New York, London, 1963).

[2] M. D. Kaplan and B. G. Vekhter, Cooperative Phenomena in Jahn-Teller Crystals (Plenum, New York, 1995).

[3] K. I. Kugel and D. I. Khomskii, Sov. Phys. - Uspekhi 25, 231 (1982).

[4] D. I. Khomskii and G. Sawatzky, Solid State. Comm. 102, 87 (1997).

[5] D. I. Khomskii, chapter in Spin Electronics, eds. M. Ziese and M. J. Thornton, (Springer, Berlin, 2001), p. 89.

[6] P. G. Radaelli, D. E. Cox, L. Capogna, S.-W. Cheong, and M. Marezio, Phys. Rev. B 59, 14440 (1999).

[7] B. Raveau, M. Hervieu, A. Maignan, and C. Martin, J. Mater. Chem. 11, 29 (2001).

[8] S. Mori, C. H. Chen, and S.-W. Cheong, Nature (London) 392, 473 (1998).

[9] T. Egami and D. Louca, Phys. Rev. B 59, 6193 (1999).

[10] D. I. Khomskii, cond-mat/0004034 (2000); J. van den Brink and D. I. Khomskii, Phys. Rev. B 63, 140416 (2001).

[11] R. Englman, The Jahn-Teller Effect in Molecules and Crystals (Wiley-Interscience, New York, 1972).

[12] R. Englman and B. Halperin, Phys. Rev. B 2, 75 (1970); B. Halperin and R. Englman, Phys. Rev. B 3, 1698 (1971)

[13] K. I. Kugel and D. I. Khomskii, JETP Lett. 15, 446 (1972); K. I. Kugel and D. I. Khomskii, Sov. Phys. JETP 37, 725 (1973).

[14] D. I. Khomskii and K. I. Kugel, Europhys. Lett. 55, 208 (2001).

[15] J. D. Eshelby, The continuum theory of lattice defects in Solid State Physics, eds. F. Seitz and D. Turnbull, 3, 79 (Academic Press, New York, 1956).

[16] M. V. Eremin, A. Yu. Zavidonov, and B. I. Kochelaev, Zh. Eksp. Teor. Fiz. 90, 537 (1986) [Sov. Phys. - JETP 63, 312 (1986)].

[17] M. A. Ivanov, V. Ya. Mitrofanov, and A. Ya. Fishman, Fiz. Tv. Tela (Sov.Phys. - Solid State) 20, 3023 (1978).

[18] J. Kanamori, J. Appl. Phys. (Suppl.) 31, 14S (1960).

[19] D. I. Khomskii and J. van den Brink, Phys. Rev. Lett. 85, 3329 (2001).

[20] Y. Yto and J. Akimitsu, J. Phys. Soc. Japan 40, 1333 (1976).

[21] D. I. Khomskii and K. I. Kugel, Solid State Comm. 13, 763 (1973).

[22] M. T. Hutchins, M. J. Fair, P. Day, and P. J. Walker, J. Phys C 9, L55 (1976). 
[23] T. G. Perring, D. T. Androja, G. Chaboussant, G. Aeppli, T. Kimura, and Y. Tokura, Phys. Rev. Lett. 82, 217201 (2001).

[24] P. Novak, J. Phys. Chem. Solids 30, 2357 (1969); 31, 125 (1970).

[25] T. Mizokawa, D. I. Khomskii, and G. A. Sawatzky, Phys. Rev. B 60, 7309 (1999).

[26] H. Kawano, R. Kajumoto, M. Kubota, and H. Yoshizawa, Phys. Rev. B 53, 2202 (1996); 53, R14709 (1996)

[27] S. Uhlenbruck, R. Teipen, R. Klingeler, B. Büchner, O. Friedt, M. Hücker, H. Kierspel, T. Niemöller, L. Pinsard, A. Revcolevschi, and R. Gross, Phys. Rev. Lett. 82, 185 (1999)

[28] Y. Yamada, O. Hino, S. Nohdo, R. Kanao, T. Inami, and S. Katano, Phys. Rev. Lett. 77, 904 (1996).

[29] T. Mizokawa, D. I. Khomskii, and G. A. Sawatzky, Phys. Rev. B 61, R3776 (2000).

[30] R. Kilian and G. Khaliullin, Phys. Rev. B 60, 13458 (1999).

[31] T. Mizokawa, D. I. Khomskii, and G. A. Sawatzky, Phys. Rev. B 63, 024403 (2001).

[32] K. H. Ahn and A. J. Millis, Phys. Rev. B 58, 3697 (1998).

[33] Y. Tomioka, A. Asamitsu, H. Kawahara, Y. Moritomo, and Y. Tokura, Phys. Rev. B 53, 1689 (1996).

[34] P. G. Radaelli, D. E. Cox, M. Marezio, and S.W. Cheong, Phys. Rev. B 55, 3015 (1997).

[35] M. v. Zimmermann, C. S. Nelson, J. P. Hill, D. Gibbs, M. Blume, D. Casa, B. Keimer, Y. Murakami, C.-C. Kao, C. Venkataraman, T. Gog, Y. Tomioka, and Y. Tokura, Phys. Rev. B 64, 195133 (2001).

[36] J. P. Hill, C. S. Nelson, M. v. Zimmermann, Y.-J. Kim, D. Gibbs, D. Casa, B. Keimer, Y. Murakami, C. Venkataraman, T. Gog, Y. Tomioka, Y. Tokura, V. Kiryukhin, T. Y. Koo, and S.-W. Cheong, Appl. Phys. A 73, 723 (2001); cond-mat/0105064.

[37] J. van den Brink, G. Khaliullin, and D. I. Khomskii, Phys. Rev. Lett. 83, 5118 (1999).

[38] E. O. Wollan and W. C. Koehler, Phys. Rev. 100, 545 (1955).

[39] Z. Jirák, S. Krupička, Z. Šimša, M. Dlouhá, and S. Vratislav, J. Magn. Magn. Mater. 53, 153 (1985).

[40] A. G. Khachaturyan, Theory of Phase Transformations and the Structure of Solid Solutions (Nauka, Moscow, 1974).

[41] M. Yu. Kagan, K. I. Kugel, and D. I. Khomskii, Zh. Exp. Teor. Fiz. 120, 470 (2001) [JETP 93, 415 (2001)]; condmat/0001245.

[42] L. D. Landau and E. M. Lifshitz, The Classical Theory of Fields (Butterworth-Heinemann, Oxford, 1997).

[43] S. J. Glass and J. O. Lawson, Phys. Lett. A 46, 234 (1973).

[44] N. Mermin and H. Wagner, Phys. Rev. Lett. 171133 (1966).

[45] K. I. Kugel and D. I. Khomskii, Fiz. Tv. Tela (Sov. Phys. - Solid State) 15, 2230 (1973).

[46] A. I. Liechtenstein, V. I. Anisimov, and J. Zaanen, Phys. Rev. B 52, R5467 (1995). 